Research Article

\title{
Comparative Study of Some Fixed-Point Methods in the Generation of Julia and Mandelbrot Sets
}

\author{
Hao Zhou, ${ }^{1}$ Muhammad Tanveer ${ }^{D},{ }^{2}$ and Jingjing $\mathrm{Li}^{3}$ \\ ${ }^{1}$ Transportation School of Wuhan University of Technology, Wuhan University of Technology, Wuhan 430070, China \\ ${ }^{2}$ Department of Mathematics and Statistics, The University of Lahore, Lahore, Pakistan \\ ${ }^{3}$ China Ship Development and Design Center, Wuhan 430064, China
}

Correspondence should be addressed to Muhammad Tanveer; tanveer.7955180@yahoo.com

Received 22 May 2020; Accepted 18 June 2020; Published 20 July 2020

Academic Editor: Basil K. Papadopoulos

Copyright (C) 2020 Hao Zhou et al. This is an open access article distributed under the Creative Commons Attribution License, which permits unrestricted use, distribution, and reproduction in any medium, provided the original work is properly cited.

Fractal is a geometrical shape with property that each point of the shape represents the whole. Having this property, fractals procured the attention in computer graphics, engineering, biology, mathematics, physics, art, and design. The fractals generated on highest priorities are the Julia and Mandelbrot sets. So, in this paper, we develop some necessary conditions for the convergence of sequences established for the orbits of $M, M^{*}$, and $K$-iterative methods to generate these fractals. We adjust algorithms according to the develop conditions and draw some attractive Julia and Mandelbrot sets with sequences of iterates from proposed fixed-point iterative methods. Moreover, we discuss the self-similarities with input parameters in each graph and present the comparison of images with proposed methods.

\section{Introduction}

The Latin word fractal (means fractured, divided, or broken) is commonly used for an image having the property of selfsimilarity in complex graphics [1]. Fractals have many applications in social sciences and engineering. In computer engineering, fractals are used to establish the security system, computer networking, image encryption, image compression, and cryptography [2]. In biology, fractals are used to study the culture of microorgans, nerve system, etc. [3]. In physics, fractals are used in fluid mechanics to understand the nature of fluids and their properties. Fractals are used in electrical and electronics engineering (i.e., in the fabricating of antennae, radar system, capacitors, security control system, radio, and antennae for wireless system) $[4,5]$. Moreover, architectural patterns and designs are also fractals [6]. Fractals have application in many other emerging fields [7-9].

Before the invention of computer, the researchers sketched aesthetic patterns, images, graphs, and geometries manually. The graph of cantor set, Koch snowflake, and Sierpinski's triangles are the patterns that can be generated manually. In 1918, Gaston Julia and Pierre Fatou defined two complementary sets (i.e., Julia set and Fatou set). But they could not sketch the graphs of Julia set and Fatou set. After the invention of computers, Mandelbrot made it possible to draw the graphs of Julia set with help of computers in 1970. He studied the Julia set for a polynomial $Q_{a_{0}}\left(z_{m}\right)=z_{m}^{2}+a_{0}$, where $z$ is a complex variable and $a_{0}$ is a complex parameter. Mandelbrot presented the characteristics of Julia set in [10] and explained that Julia set had great diversity of aesthetic designs [11]. The Mandelbrot set for $Q_{a_{0}}\left(z_{m}\right)=z_{m}^{p}+a_{0}$, where $z$ is a complex variable and $a_{0}$ is a complex parameter, was discussed in [12]. The images resembled with Julia and Mandelbrot sets for rational and transcendental complex functions were visualized in [13]. Some 4D and 3D fractals for quaternions and bicomplex and tricomplex functions were studied in $[14,15]$ and [16]. To generalize Julia and Mandelbrot sets, initially Rani et al. used fixed-point theory in the generation of fractals (refer in $[17,18]$ ). Some generalized fractals via explicit fixed-point iterative methods were studied in [19-24]. The implicit iterative methods were used to develop convergence criterion for fractals in [25-30]. There are many fixed-point methods that can be used for fractal generation [31-36]. 
There are some well-known criterions to generate the fractals such as distance estimator [37], potential function algorithms [38], and escape criteria [39]. In this paper, we use escape criterion conditions to sketch some bewitching Julia and Mandelbrot sets. In this paper, we develop some necessary conditions for the convergence of $\left|Q_{a_{0}}^{m}\right|$ to generate fractals (i.e., especially for Julia and Mandelbrot sets) via some fixed-point iterative methods. We use proposed conditions in algorithms to sketch Julia and Mandelbrot sets. Furthermore, we present some graphs to compare the images. The influence of input parameters on images is also discussed.

This paper is composed of five sections: Section 2 deals with some basic concepts about fractals and fixed-point iterative methods; in Section 3, we develop some convergence conditions to generate fractals; we establish comparison among Julia sets and Mandelbrot sets via proposed methods in Section 4, and at the end, we conclude this paper in Section 5.

\section{Some Basic Concepts}

In this section, we discuss some basic concepts.

Definition 1 (Julia set [40]). Let $Q_{a_{0}}\left(z_{m}\right)=z_{m}^{p}+a_{0}$ be a complex polynomial with $p \geq 2$. Then, the set of points $J_{Q_{a_{0}}}$ in $\mathbb{C}$ is named as the filled Julia set, when the orbits of the points in $J_{Q_{a_{0}}}$ does not move to $\infty$ as $m \longrightarrow \infty$, i.e.,

$$
J_{Q_{a_{0}}}=\left\{z \in \mathbb{C}:\left\{\left|Q_{a_{0}}^{m}\right|\right\}_{m=0}^{\infty} \text { is bounded }\right\},
$$

where $Q_{a_{0}}^{m}$ is the $m$ - th iterate of $z$. The set of boundary points of $J_{Q_{a_{0}}}$ is called the simple Julia set.

Definition 2 (Mandelbrot set [41]). The collection of all connected Julia sets is defined as the Mandelbrot set $M$, i.e.,

$$
M=\left\{a_{0} \in \mathbb{C}: J_{Q_{a_{0}}} \text { is connected }\right\} .
$$

Equivalently, the Mandelbrot set is defined as [42]

$$
M=\left\{a_{0} \in \mathbb{C}:\left\{Q_{a_{0}}^{m}(0)\right\} \nrightarrow \infty \text { as } m \longrightarrow \infty\right\} .
$$

Since the critical point of $Q_{a_{0}}$ is 0 , so the authors set $z_{0}=0$ as an initial guess. There are many fixed-point iterative methods in literature that can be used to generate fractals. For each method, the authors prove escape criterion to generate fractals. In this paper, we use $M, M^{*}$, and $K$ iterative methods to visualize Julia and Mandelbrot sets. The proposed fixed-point iterative methods are defined as follows.

Definition 3 (M-iterative method [43]). Let $Q: \mathbb{C} \longrightarrow \mathbb{C}$ be a complex polynomial with $p \geq 2$. For any $z_{0} \in \mathbb{C}$, the $M$-iterative method is defined as

$$
\left\{\begin{array}{l}
z_{m+1}=Q\left(w_{k}\right), \\
w_{m}=Q\left(u_{m}\right), \\
u_{m}=(1-a) z_{m}+a Q\left(z_{m}\right),
\end{array}\right.
$$

where $a \in(0,1]$ and $m=0,1,2, \ldots$

Definition $4\left(M^{*}\right.$-iterative method [43]). Let $Q: \mathbb{C} \longrightarrow \mathbb{C}$ be a complex polynomial with $p \geq 2$. For any $z_{0} \in \mathbb{C}$, the $M^{*}$-iterative method is defined as

$$
\left\{\begin{array}{l}
z_{m+1}=Q\left(w_{k}\right), \\
w_{m}=Q\left(v_{m}\right), \\
u_{m}=(1-a) z_{m}+a Q\left(z_{m}\right),
\end{array}\right.
$$

where $\quad v_{m}=(1-b) z_{m}+b Q\left(u_{m}\right), a, b \in(0,1], \quad$ and $m=0,1,2, \ldots$.

Definition 5 (K-iterative method [43]). Let $Q: \mathbb{C} \longrightarrow \mathbb{C}$ be a complex polynomial with $p \geq 2$. For any $z_{0} \in \mathbb{C}$, the $M^{*}$-iterative method is defined as

$$
\left\{\begin{array}{l}
z_{m+1}=Q\left(w_{k}\right), \\
w_{m}=Q\left(v_{m}\right), \\
u_{m}=(1-a) z_{m}+a Q\left(z_{m}\right),
\end{array}\right.
$$

where $\quad v_{m}=(1-b) Q\left(z_{m}\right)+b Q\left(u_{m}\right), a, b \in(0,1], \quad$ and $m=0,1,2, \ldots$..

The sequence of iterates $\left\{z_{m}\right\}_{m \in \mathbb{N}}$ defined by (4)-(6) is called the orbit.

\section{Convergence Analysis}

Here, we prove some convergence conditions (i.e., escape criterion) for complex polynomial $Q_{a_{0}}(z)=z^{p}+a_{0}$, where $p \geq 2$ and $a_{0} \in \mathbb{C}$ via $M, M^{*}$, and $K$-iterative methods, respectively. Without necessary conditions, we cannot generate fractal because the convergence condition is the basic key to run the algorithm. Throughout this section, we use $Q(z)$ as $Q_{a_{0}}(z)$ and $z_{0}=z, u_{0}=u, v_{0}=v$, and $w_{0}=w$ in the following way.

Theorem 1. Let $Q_{a_{0}}=z^{p}+a_{0}$ be a complex polynomial with $|z| \geq\left|a_{0}\right|>(2 / a)^{\left(1 / p^{-1)}\right.}$ and $|z| \geq\left|a_{0}\right|>(2 / b)^{(1 / p-1)}$, where $p \geq 2, a, b \in(0,1]$, and $a_{0} \in \mathbb{C}$. The sequence of iterates $\left\{z_{m}\right\}_{m \in \mathbb{N}}$ for the K-iterative method is defined as follows:

$$
\left\{\begin{array}{l}
z_{m+1}=Q\left(w_{k}\right), \\
w_{m}=Q\left(v_{m}\right), \\
u_{m}=(1-a) z_{m}+a Q\left(z_{m}\right),
\end{array}\right.
$$

where $v_{m}=(1-b) Q\left(z_{m}\right)+b Q\left(u_{m}\right), a, b \in(0,1]$, and $m=0,1,2, \ldots$. Then, $\left|z_{m}\right| \longrightarrow \infty$ as $m \longrightarrow \infty$.

Proof. Because $Q(z)=z^{p}+a_{0}$, where $a_{0} \in \mathbb{C}, z_{0}=z$, $u_{0}=u, v_{0}=v$, and $w_{0}=w$, then, for first step of the $K$-iterative method, we have

$$
u_{m}=(1-a) z_{m}+a \mathrm{Q}\left(z_{m}\right) \text {. }
$$

For $m=0$, we have 


$$
\begin{aligned}
\left|u_{0}\right| & =|(1-a) z+a Q(z)| \\
& =\left|(1-a) z+a\left(z^{p}+a_{0}\right)\right| \\
\left|u_{0}\right| & \geq a\left|z^{2}+a_{0}\right|-(1-a)|z| \\
& \geq a\left|x^{2}\right|-a\left|a_{0}\right|-|z|+|z| \\
& \geq a\left|z^{2}\right|-|z|, \quad \because|z| \geq\left|a_{0}\right| \\
\left|u_{0}\right| & =|z|(a|z|-1) .
\end{aligned}
$$

Since $\quad|z| \geq\left|a_{0}\right|>(2 / a)^{(1 / p-1)}, \quad$ this yields $\left|z^{p}\right|\left(a\left|z^{p-1}\right|-1\right)^{p}>\left|z^{p}\right| \geq a\left|z^{p}\right|$. Thus, $\left|u^{p}\right| \geq a\left|z^{p}\right|$.

For second step of $K$-iteration, we have

$$
w_{m}=Q\left(v_{m}\right) \text {, }
$$

where $v_{m}=(1-b) Q\left(z_{m}\right)+b Q\left(u_{m}\right)$. For $m=0$, we get

$$
\begin{aligned}
\left|w_{0}\right| & =\left|Q\left(v_{0}\right)\right| \\
& =\left|v^{p}+a_{0}\right| .
\end{aligned}
$$

Thus,

$$
\left|w_{0}\right| \geq\left|v^{p}\right|-\left|a_{0}\right|
$$

Since $v_{m}=(1-b) Q\left(z_{m}\right)+b Q\left(u_{m}\right)$, then

$$
\begin{aligned}
\left|v_{0}\right| & =\left|(1-b) Q\left(z_{0}\right)+b Q\left(u_{0}\right)\right| \\
\left|v_{0}\right| & =\left|(1-b)\left(z^{p}+a_{0}\right)+b\left(u^{p}+a_{0}\right)\right| \\
& \geq b\left|u^{p}\right|-b\left|a_{0}\right|-(b-1)\left|z^{p}\right|-(1-b)\left|a_{0}\right| \\
& \geq a b\left|z^{p}\right|-(b-1)\left|z^{p}\right|-\left|a_{0}\right|, \quad \because\left|u^{p}\right| \geq a\left|z^{p}\right| \\
& \geq(a b-b+1)\left|z^{p}\right|-|z|, \quad \because|z|>\left|a_{0}\right| \\
& \geq a b\left|z^{p}\right|-|z|, \quad \because a b+(1-b)>a b \\
\left|v_{0}\right| & =|z|\left(a b\left|z^{p-1}\right|-1\right) .
\end{aligned}
$$

Since $|z|>(2 / a)^{(1 / p-1)}$ and $|z|>(2 / b)^{(1 / p-1)}$, this yields $|z|>(2 / a b)^{(1 / p-1)}$. Following this, we get $a b\left|z^{p-1}\right|-1>1$ and $\left|z^{p}\right|\left(a b\left|z^{p-1}\right|-1\right)^{p}>\left|z^{p}\right|>a b\left|z^{p}\right|$. Therefore,

$$
\left|v_{0}^{p}\right|>a b\left|z^{p}\right| .
$$

From (12) and (14), we have

$$
\begin{aligned}
\left|w_{0}\right| & \geq a b\left|z^{p}\right|-\left|a_{0}\right| \\
& \geq|z|\left(a b\left|z^{p-1}\right|-1\right), \quad \because|z|>\left|a_{0}\right| .
\end{aligned}
$$

It follows that

$$
\left|w_{0}^{p}\right|>a b\left|z^{p}\right|
$$

The last step of the $\mathrm{K}$-iterative method is

$$
z_{m+1}=Q\left(w_{m}\right) .
$$

For $m=0$, we have

$$
\begin{aligned}
\left|z_{1}\right| & =\left|Q\left(w_{0}\right)\right| \\
& =\left|w^{p}+a_{0}\right| \\
& \geq\left|w^{2}\right|-\left|a_{0}\right| \\
z_{1} & \geq a b\left|z^{p}\right|-|z|, \quad \because|z|>\left|a_{0}\right| .
\end{aligned}
$$

From (17),

$$
\left|z_{1}\right| \geq|z|\left(a b\left|z^{p-1}\right|-1\right) .
$$

Since $|z|>(2 / a)^{(1 / p-1)}$ and $|z|>(2 / b)^{(1 / p-1)}$, then $|z|>(2 / a b)^{(1 / p-1)}$, this implies $a b\left|z^{p-1}\right|-1>1$. Thus, there exists positive number $\eta>0$ such that $a b\left|z^{p-1}\right|-1>1+\eta$, which yields $\left|z_{1}\right|>(1+\eta)|z|$. Particularly, $\left|z_{1}\right|>|z|$. Subsequently, $\quad\left|z_{m}\right|>(1+\eta)^{m}|z|$. Hence, $\quad\left|z_{m}\right| \longrightarrow \infty$ as $m \longrightarrow \infty$.

Corollary 1. Suppose that

$$
\begin{aligned}
& \left|a_{0}\right|>\left(\frac{2}{a}\right)^{1 / p-1} \\
& \left|a_{0}\right|>\left(\frac{2}{b}\right)^{1 / p-1}
\end{aligned}
$$

then the orbit of the K-iterative method escapes to infinity.

Corollary 2. Suppose that $a, b \in(0,1]$ and

$$
|z|>\max \left[\left|a_{0}\right|,\left(\frac{2}{a}\right)^{(1 / p-1)},\left(\frac{2}{b}\right)^{(1 / p-1)}\right],
$$

therefore, there exists $\eta>0$ such that $\left|z_{m}\right|>(1+\lambda)^{m}|z|$ and $\left|z_{m}\right| \longrightarrow \infty$ as $m \longrightarrow \infty$.

Corollary 3. Assume that

$$
\left|z_{k}\right|>|z|>\max \left[\left|a_{0}\right|,\left(\frac{2}{a}\right)^{(1 / p-1)},\left(\frac{2}{b}\right)^{(1 / p-1)}\right]
$$

for some $k \geq 0$. Thus, there exists $\eta>0$ such that $\left|z_{k+m}\right|>(1+\lambda)^{m}\left|z_{m}\right|$ and $\left|z_{m}\right| \longrightarrow \infty$ as $m \longrightarrow \infty$.

Theorem 2. Let $Q_{a_{0}}=z^{p}+a_{0}$ be a complex polynomial with $\left.|z| \geq\left|a_{0}\right|>(2 / a)^{\left(1 / p^{-1}\right.} 1\right)$ and $|z| \geq\left|a_{0}\right|>(2 / b)^{(1 / p-1)}$, where $p \geq 2, a, b \in(0,1]$, and $a_{0} \in \mathbb{C}$. The sequence of iterates $\left\{z_{m}\right\}_{m \in \mathbb{N}}$ for the $M^{*}$-iterative method is defined as follows:

$$
\left\{\begin{array}{l}
x_{m+1}=Q\left(w_{k}\right), \\
w_{m}=Q\left(v_{m}\right), \\
u_{m}=(1-a) z_{m}+a Q\left(z_{m}\right),
\end{array}\right.
$$

where $\quad v_{m}=(1-b) z_{m}+b Q\left(u_{m}\right), a, b \in(0,1], \quad$ and $m=0,1,2, \ldots$. Then, $\left|z_{m}\right| \longrightarrow \infty$ as $m \longrightarrow \infty$.

Proof. Because $Q(z)=z^{p}+a_{0}$, where $a_{0} \in \mathbb{C}, z_{0}=z$, $u_{0}=u, v_{0}=v$, and $w_{0}=w$, then, for first step of the $M^{*}$-iterative method, we have

$$
u_{m}=(1-a) z_{m}+a \mathrm{Q}\left(z_{m}\right) .
$$

For $m=0$, we have 


$$
\begin{aligned}
\left|u_{0}\right| & =|(1-a) z+a Q(z)| \\
& =\left|(1-a) z+a\left(z^{p}+a_{0}\right)\right| \\
\left|u_{0}\right| & \geq a\left|z^{p}+a_{0}\right|-(1-a)|z| \\
& \geq a\left|z^{p}\right|-a\left|a_{0}\right|-|z|+|z| \\
& \geq a\left|z^{p}\right|-|z|, \quad \because|z| \geq\left|a_{0}\right| \\
\left|u_{0}\right| & =|z|\left(a\left|z^{p-1}\right|-1\right) .
\end{aligned}
$$

Since $|z| \geq\left|a_{0}\right|>(2 / a)^{(1 / p-1)}$, this creates the situation $|z|\left(a\left|z^{p-1}\right|-1\right)>|z| \geq a|z|$. Thus, $\left|u^{p}\right| \geq a\left|z^{p}\right|$.

For the second step of the $M^{*}$-iterative method, we have

$$
w_{m}=Q\left(v_{m}\right),
$$

where $v_{m}=(1-b) Q\left(z_{m}\right)+b Q\left(u_{m}\right)$. For $m=0$, we get

$$
\begin{aligned}
\left|w_{0}\right| & =\left|Q\left(v_{0}\right)\right| \\
& =\left|v^{p}+a_{0}\right| .
\end{aligned}
$$

Thus,

$$
\left|w_{0}\right| \geq\left|v^{p}\right|-\left|a_{0}\right|
$$

Since $v_{m}=(1-b) z_{m}+b Q\left(u_{m}\right)$, then

$$
\begin{aligned}
\left|v_{0}\right| & =\left|(1-b) z_{0}+b Q\left(u_{0}\right)\right| \\
\left|v_{0}\right| & =\left|(1-b) z+b\left(u^{p}+a_{0}\right)\right| \\
& \geq b\left|u^{p}\right|-b\left|a_{0}\right|-(1-b)|z| \\
& \geq a b\left|z^{p}\right|-(1-b)|z|-b\left|a_{0}\right|, \quad \because\left|u^{p}\right| \geq a\left|z^{p}\right| \\
& \geq a b\left|z^{p}\right|-|z|+b|z|-b|z|, \quad \because|z|>\left|a_{0}\right| \\
& =a b\left|z^{p}\right|-|z|\left|v_{0}\right| \\
& =|z|\left(a b\left|z^{p-1}\right|-1\right) .
\end{aligned}
$$

Since $|z|>(2 / a)^{(1 / p-1)}$ and $|z|>(2 / b)^{(1 / p-1)}$, this yields $.|z|>(2 / a b)^{(1 / p-1)}$. Following this, we get $a b\left|z^{p-1}\right|-1>1$ and $\left|z^{p}\right|\left(a b\left|z^{p-1}\right|-1\right)^{p}>\left|z^{p}\right|>a b\left|z^{p}\right|$. Therefore,

$$
\left|v_{0}^{p}\right|>a b\left|z^{p}\right| \text {. }
$$

From (28) and (30), we have

$$
\begin{aligned}
\left|w_{0}\right| & \geq a b\left|z^{p}\right|-\left|a_{0}\right| \\
& \geq|z|\left(a b\left|z^{p-1}\right|-1\right), \quad \because|z|>\left|a_{0}\right| .
\end{aligned}
$$

It follows that

$$
\left|w_{0}^{p}\right|>a b\left|z^{p}\right| .
$$

The last step of the $M^{*}$-iterative method is

$$
z_{m+1}=Q\left(w_{m}\right) .
$$

For $m=0$ and using (32), we have

$$
\begin{aligned}
\left|z_{1}\right| & =\left|Q\left(w_{0}\right)\right| \\
& =\left|w^{p}+a_{0}\right| \\
& \geq\left|w^{p}\right|-\left|a_{0}\right| \\
z_{1} & \geq a b\left|z^{p}\right|-|z|, \quad \because|z|>\left|a_{0}\right| .
\end{aligned}
$$

Therefore,

$$
\left|z_{1}\right| \geq|z|\left(a b\left|z^{p-1}\right|-1\right) .
$$

Since $|z|>(2 / a)^{(1 / p-1)}$ and $|z|>(2 / b)^{(1 / p-1)}$, then $|z|>(2 / a b)^{(1 / p-1)}$, this implies $a b\left|z^{p^{-1}}\right|-1>1$. Thus, there exists positive number $\eta>0$ such that $a b\left|z^{p-1}\right|-1>1+\eta$, which yields $\left|z_{1}\right|>(1+\eta)|z|$. Particularly, $\left|z_{1}\right|>|z|$. Subsequently, $\quad\left|z_{m}\right|>(1+\eta)^{m}|z|$. Hence, $\quad\left|z_{m}\right| \longrightarrow \infty$ as $m \longrightarrow \infty$.

Corollary 4. Suppose that

$$
\begin{aligned}
& \left|a_{0}\right|>\left(\frac{2}{a}\right)^{(1 / p-1)}, \\
& \left|a_{0}\right|>\left(\frac{2}{b}\right)^{(1 / p-1)},
\end{aligned}
$$

then the orbit of the $M^{*}$-iterative method escapes to infinity.

Corollary 5. Suppose that $a, b \in(0,1]$ and

$$
|z|>\max \left[\left|a_{0}\right|,\left(\frac{2}{a}\right)^{(1 / p-1)},\left(\frac{2}{b}\right)^{(1 / p-1)}\right],
$$

therefore, there exists $\eta>0$ such that $\left|z_{m}\right|>(1+\lambda)^{m}|z|$ and $\left|z_{m}\right| \longrightarrow \infty$ as $m \longrightarrow \infty$.

Corollary 6. Assume that

$$
\left|z_{k}\right|>|z|>\max \left[\left|a_{0}\right|,\left(\frac{2}{a}\right)^{(1 / p-1)},\left(\frac{2}{b}\right)^{(1 / p-1)}\right]
$$

for some $k \geq 0$. Thus, there exists $\eta>0$ such that $\left|z_{k+m}\right|>(1+\lambda)^{m}\left|z_{m}\right|$ and $\left|z_{m}\right| \longrightarrow \infty$ as $m \longrightarrow \infty$.

Theorem 3. Let $Q_{a_{0}}=z^{p}+a_{0}$ be a complex polynomial with $|z| \geq\left|a_{0}\right|>(2 / a)^{\left(1 / p^{0}\right.}{ }^{1)}$, where $p \geq 2, a, b \in(0,1]$, and $a_{0} \in \mathbb{C}$. The sequence of iterates $\left\{z_{m}\right\}_{m \in \mathbb{N}}$ for the M-iterative method is defined as follows:

$$
\left\{\begin{array}{l}
x_{m+1}=Q\left(w_{k}\right), \\
w_{m}=Q\left(u_{m}\right), \\
u_{m}=(1-a) z_{m}+a Q\left(z_{m}\right),
\end{array}\right.
$$

where $a, b \in(0,1]$ and $m=0,1,2, \ldots$ Then, $\left|z_{m}\right| \longrightarrow \infty$ as $m \longrightarrow \infty$.

Proof. Because $Q(z)=z^{p}+a_{0}$, where $a_{0} \in \mathbb{C}, z_{0}=z$, $u_{0}=u, v_{0}=v$, and $w_{0}=w$, then, for the first of $M$-iterative method, we have

$$
u_{m}=(1-a) z_{m}+a \mathrm{Q}\left(z_{m}\right) .
$$

For $m=0$, we have 


$$
\begin{aligned}
\left|u_{0}\right| & =|(1-a) z+a Q(z)| \\
& =\left|(1-a) z+a\left(z^{p}+a_{0}\right)\right| \\
\left|u_{0}\right| & \geq a\left|z^{p}+a_{0}\right|-(1-a)|z| \\
& \geq a\left|z^{p}\right|-a\left|a_{0}\right|-|z|+|z| \\
& \geq a\left|z^{p}\right|-|z|, \quad \because|z| \geq\left|a_{0}\right| \\
\left|u_{0}\right| & =|z|\left(a\left|z^{p-1}\right|-1\right) .
\end{aligned}
$$

Since $|z| \geq\left|a_{0}\right|>(2 / a)^{(1 / p-1)}$, this creates the situation $|z|\left(a\left|z^{p-1}\right|-1\right)>|z| \geq a|z|$. Thus, $\left|u^{p}\right| \geq a\left|z^{p}\right|$.

For the second step of $M$-iteration, we have

$$
w_{m}=Q\left(u_{m}\right) \text {. }
$$

For $m=0$, we get

$$
\begin{array}{r}
\left|w_{0}\right|=\left|Q\left(u_{0}\right)\right| \\
=\left|u^{p}+a_{0}\right| .
\end{array}
$$

Thus,

$$
\begin{aligned}
\left|w_{0}\right| & =\left|Q\left(u_{0}\right)\right| \\
& =\left|u^{p}+a_{0}\right| \\
& \geq\left|u^{p}\right|-\left|a_{0}\right| \\
& \geq a\left|z^{p}\right|-|c|, \quad \because\left|u^{p}\right| \\
& \geq a\left|z^{p}\right| \geq a\left|z^{p}\right|-|z|, \quad \because|z|>\left|a_{0}\right| \\
& =a\left|z^{p}\right|-|z| \\
\left|v_{0}\right| & =|z|\left(a\left|z^{p-1}\right|-1\right) .
\end{aligned}
$$

Since $|z|>(2 / a)^{(1 / p-1)}$, it follows $a\left|z^{p-1}\right|-1>1$ and $\left|z^{p}\right|\left(a\left|z^{p-1}\right|-1\right)^{p}>\left|z^{p}\right|>a\left|z^{p}\right|$. Therefore,

$$
\left|w_{0}^{p}\right|>b\left|z^{p}\right| \text {. }
$$

In the last step of the $M$-iterative method, we have

$$
z_{m+1}=Q\left(w_{m}\right)
$$

For $m=0$, we have

$$
\begin{aligned}
\left|z_{1}\right| & =\left|Q\left(w_{0}\right)\right| \\
& =\left|w^{p}+a_{0}\right| \\
& \geq\left|w^{p}\right|-\left|a_{0}\right| \\
& \geq a\left|z^{p}\right|-|z|, \quad \because|z|>\left|a_{0}\right| .
\end{aligned}
$$

From (46),

$$
\left|z_{1}\right| \geq|z|\left(a\left|z^{p-1}\right|-1\right)
$$

Since $|z|>(2 / a)^{(1 / p-1)}$, this implies $a\left|z^{p-1}\right|-1>1$. Thus, there exists positive number $\eta>0$ such that $a\left|z^{p-1}\right|-1>1+\eta$, which yields $\left|z_{1}\right|>(1+\eta)|z|$. Particularly, $\left|z_{1}\right|>|z|$. Subsequently, $\left|z_{m}\right|>(1+\eta)^{m}|z|$. Hence, $\left|z_{m}\right| \longrightarrow \infty$ as $m \longrightarrow \infty$.

\section{Corollary 7. Suppose that}

$$
\left|a_{0}\right|>\left(\frac{2}{a}\right)^{(1 / p-1)},
$$

then the orbit of the M-iterative method escapes to infinity.

Corollary 8. Suppose that $a, b \in(0,1]$ and

$$
|z|>\max \left[\left|a_{0}\right|,\left(\frac{2}{a}\right)^{(1 / p-1)}\right],
$$

therefore, there exists $\eta>0$ such that $\left|z_{m}\right|>(1+\lambda)^{m}|z|$ and $\left|z_{m}\right| \longrightarrow \infty$ as $m \longrightarrow \infty$.

Corollary 9. Assume that

$$
\left|z_{k}\right|>|z|>\max \left[\left|a_{0}\right|,\left(\frac{2}{a}\right)^{(1 / p-1)}\right]
$$

for some $k \geq 0$. Thus, there exists $\eta>0$ such that $\left|z_{k+m}\right|>(1+\lambda)^{m}\left|z_{m}\right|$ and $\left|z_{m}\right| \longrightarrow \infty$ as $m \longrightarrow \infty$.

\section{Applications of Fractals}

To visualize the fractals, some convergence conditions are required, and actually, these are the main tools to execute the algorithm properly and sketch the desired type of fractals. In literature, the authors fixed maximum number of iterations up to hundred. To check self-similarity and get better results, we fixed the maximum number of iterations at 1000. In this section, we adjust two algorithms: one for the Julia set and other for the Mandelbrot set to generate fractals via proposed methods. We visualize some Julia and Mandelbrot sets for different involve parameters.

4.1. Julia Sets. Julia is known as the pioneer of complex fractals. In this subsection, we sketch some graphs of Julia set at different input parameters. We generate Julia sets for $M$, $M^{*}$, and $K$-iterative methods by using Algorithm 1 and compare the images of Julia set for proposed methods.

Example 1. In this example, we present the Julia sets for a polynomial $Q(z)=z^{2}+a_{0}$, where $a_{0} \in \mathbb{C}$ in the orbits of $M$, $M^{*}$, and $K$-iterative methods, respectively. The graphs in Figures $1-3$ for $a_{0}=-0.05-0.63 \mathbf{i}, a=0.01, b=0.9$, and $A=[-1.5,1.5]^{2}$ are quadratic Julia sets in the orbits of $M$, $M^{*}$, and $K$-iterative methods, respectively. The images in Figures 1 and 3 are Julia sets resembling Chinese dragon having two repelling fixed points: one is at the right end spiral, and other is in spiral on the left side. The image in Figure 2 is a filled connected quadratic Julia set. The graphs in Figures 4-6 with $a_{0}=-0.8, a=0.01, b=0.9$, and $A=$ $[-1.8,1.8] \times[-1.5,1.5]$ are in symmetry along the $x$-axis. Each image is a junction of two quadratic Mandelbrot sets having opposite directions but slightly different from each other in shape of bulbs on main body. Now for $a_{0}=0.749 \mathbf{i}$ with $a=0.01, b=0.9$, and $A=[-1.5,1.5] \times[-1.3,1.3]$, we notice the graphs have quite different shapes, as shown in Figures 7-9. The images in Figures 7 and 8 resemble the lighting in sky, while the image in Figure 8 has two big and many small spirals. 
Input: $Q_{a_{0}}=z^{p}+a_{0}-\mathrm{a}$ complex polynomial, $A$-covered area, $\left.M=1000, a, b \in(0,1]\right), a_{0} \in \mathbb{C}$-involved parameters, coloursmap $[0 . . h-1]$ with $h$ colours.

Output: sketched Julia set.

(1) for $z_{0} \in A$ do

(2) $R$-convergence condition for proposed method

(3) $m=0$

(4) while $m \leq M$ do

(5) Proposed iterative method

(6) if $\left|z_{m+1}\right|>R$ then

(7) break

(8) $\quad m=m+1$

(9) $\quad i=\lfloor(h-1) m / M\rfloor$

(10) colour $z_{0}$ with coloursmap $[i]$

Algorithm 1: Pseudocode for Julia set.

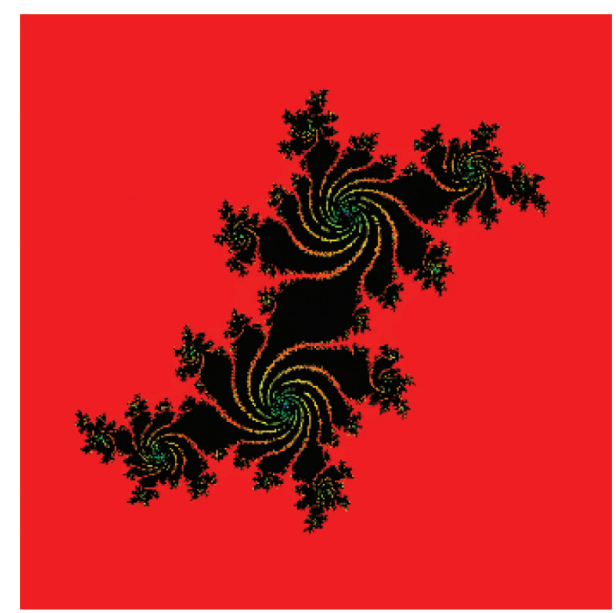

Figure 1: Julia set for $Q(z)$ with $n=2$ in $M$-orbit.

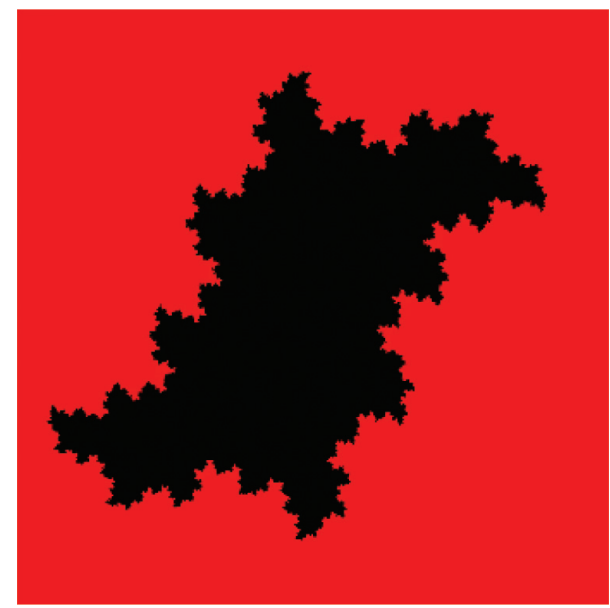

FIGURE 2: Julia set for $Q(z)$ with $n=2$ in $M^{*}$-orbit.

Example 2. In second example, we visualize some cubic Julia sets for a polynomial $Q(z)=z^{3}+a_{0}$, where $a_{0} \in \mathbb{C}$ in the orbits of $M, M^{*}$, and $K$-iterative methods, respectively. The images in Figures 10-12 are like the cubic Douady rabbits.

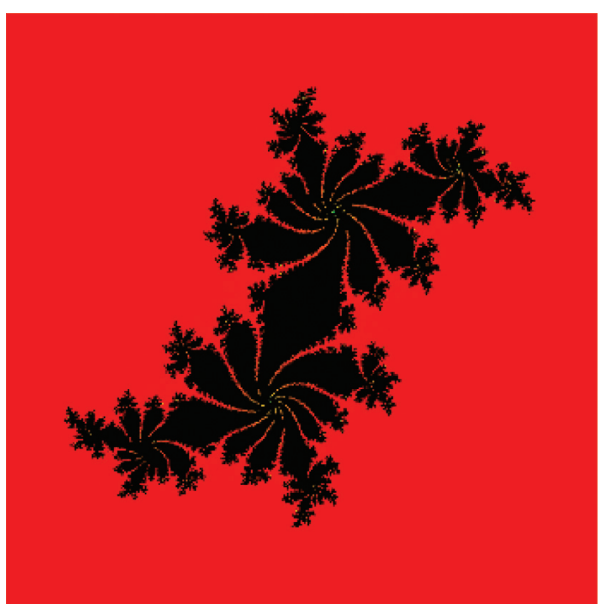

Figure 3: Julia set for $Q(z)$ with $n=2$ in $K$-orbit.

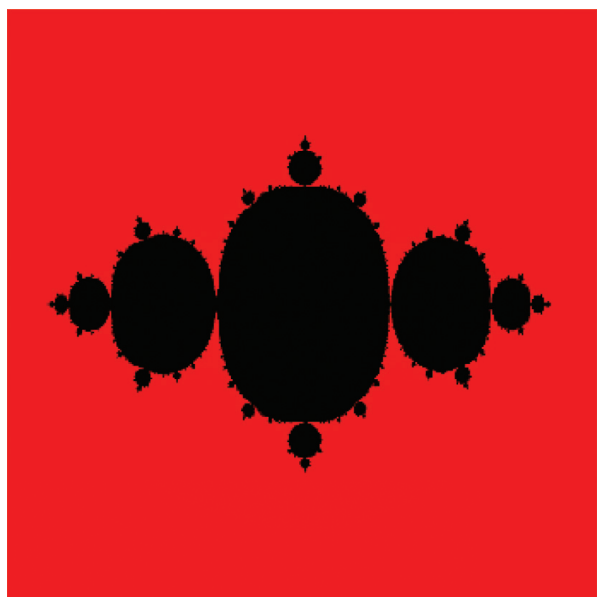

FIgURE 4: Julia set for $Q(z)$ with $n=2$ in $M$-orbit.

We observe the image in Figure 10 is a smart Douady rabbit, in Figure 11 is a fat Douady rabbit, and in Figure 12 is a relatively weak but more attractive Douady rabbit for cubic complex polynomial. The main body of graphs in Figures $13-15$ is like a circular saw having three teeth. The 


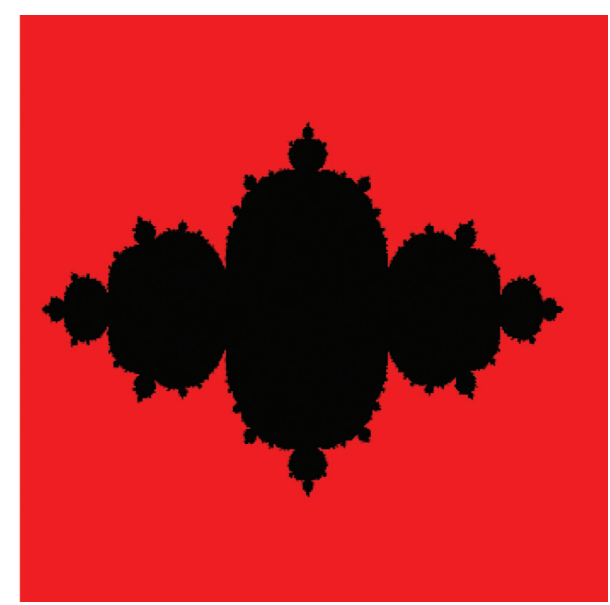

FIgURE 5: Julia set for $Q(z)$ with $n=2$ in $M^{*}$-orbit.

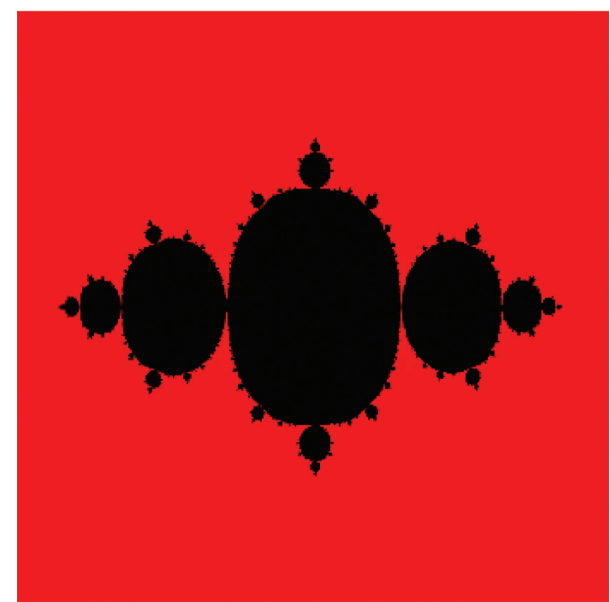

FIgURE 6: Julia set for $Q(z)$ with $n=2$ in $K$-orbit.

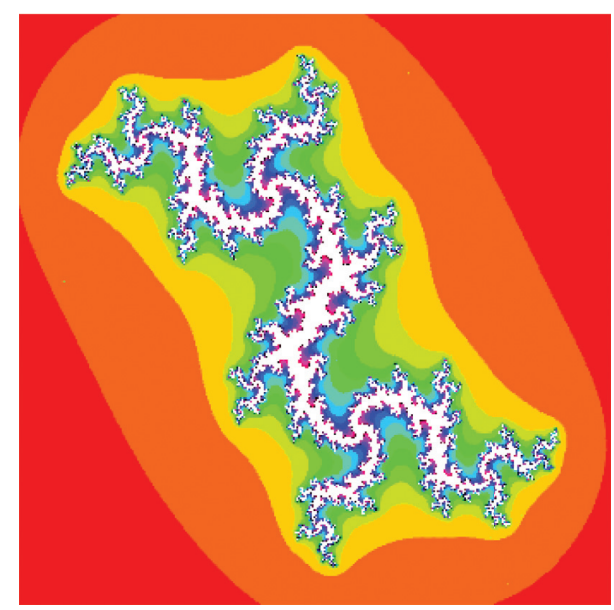

FIgURE 7: Julia set for $Q(z)$ with $n=2$ in $M$-orbit.

curl shape top of each teeth of the main saw is joint with the teeth of small circular saw and so on. Each image in Figures 13-15 have a main circular saw-type body with three large saws and six small saws. The saws for $M$ and $K$ methods

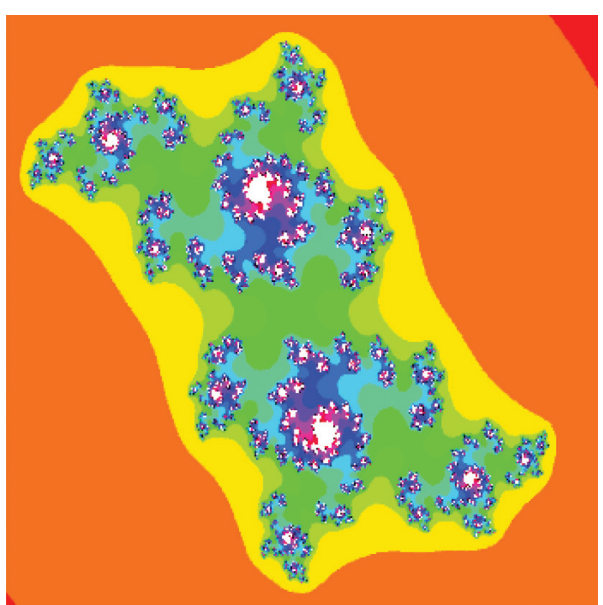

FIgURE 8: Julia set for $Q(z)$ with $n=2$ in $M^{*}$-orbit.

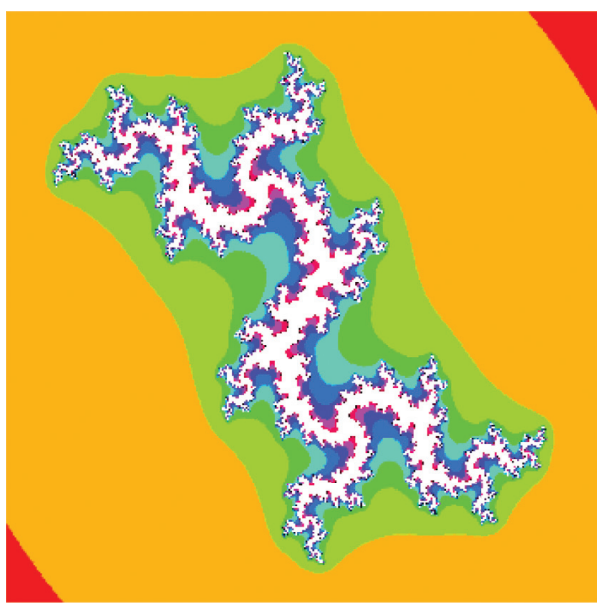

Figure 9: Julia set for $Q(z)$ with $n=2$ in $K$-orbit.

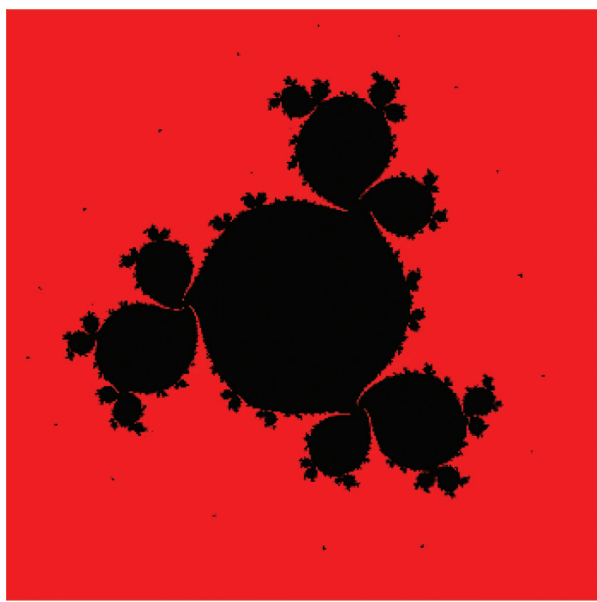

FIgURE 10: Julia set for $Q(z)$ with $n=3$ in $M$-orbit.

are sharp, while saw for $M^{*}$ is blunt. The input parameters are as follows:

(i) Figures 10-12 have input parameters $a_{0}=0.5+0.5 \mathbf{i}$, $a=0.01, b=0.9$, and $A=[-1.5,1.5]^{2}$ 


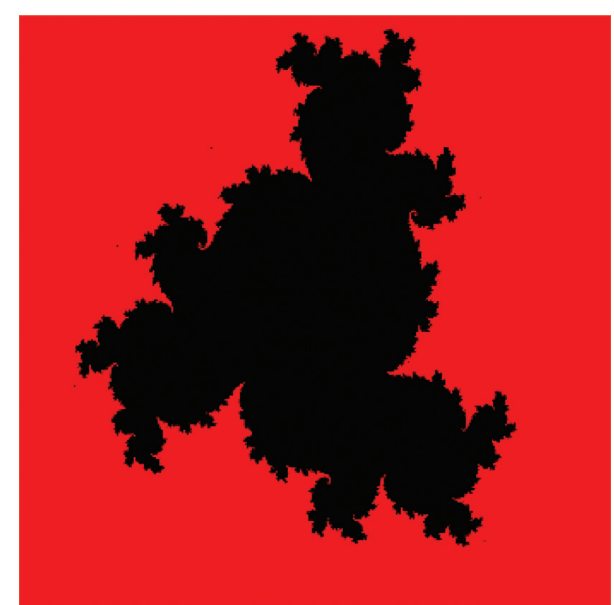

Figure 11: Julia set for $Q(z)$ with $n=3$ in $M^{*}$-orbit.

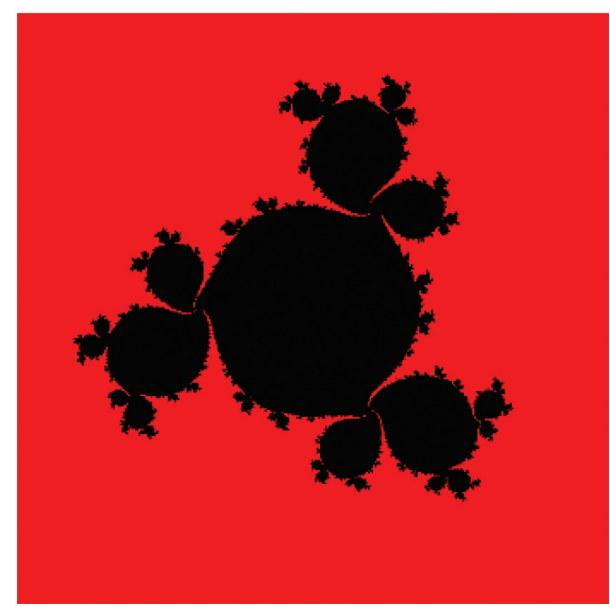

Figure 12: Julia set for $Q(z)$ with $n=3$ in $K$-orbit.

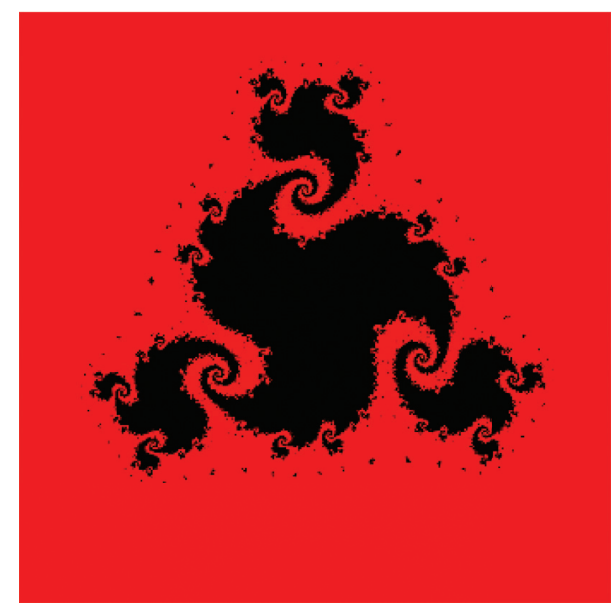

FIGURE 13: Julia set for $Q(z)$ with $n=3$ in $M$-orbit.

(ii) Figures $13-15$ are $a_{0}=-0.1+0.8 \mathbf{i}, \quad a=0.9, b=$ 0.005 , and $A=[-1.5,1.5]^{2}$

Example 3. This example presents some biquadratic Julia sets for a polynomial $Q(z)=z^{4}+a_{0}$, where $a_{0} \in \mathbb{C}$ in the

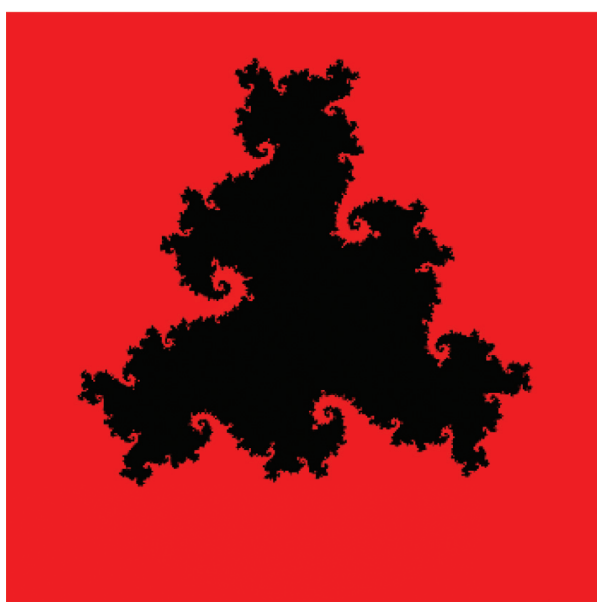

FIgURE 14: Julia set for $Q(z)$ with $n=3$ in $M^{*}$-orbit.

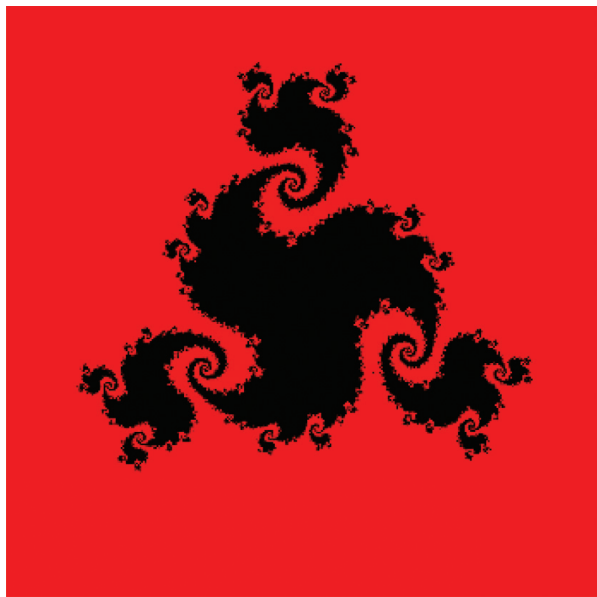

Figure 15: Julia set for $Q(z)$ with $n=3$ in $K$-orbit.

orbits of $M, M^{*}$, and $K$-iterative methods, respectively. The image in Figure 16 has a variety of colours. The images in Figures 16-18 are disconnected Julia sets and different in shapes at the same inputs $a_{0}=-0.8-0.3 \mathbf{i}, a=0.7, b=0.7$, and $A=[-1.5,1.5]^{2}$.

4.2. Mandelbrot Sets. Mandelbrot examined the graph of complex polynomial $Q(z)=z^{2}+a_{0}$ and observed that the main body of image is a cardioid having a large bulb symmetry along $x$-axis and two small bulbs symmetry along $y$-axis. The image of $Q(z)$ is usually called the classical Mandelbrot set, and it is also called God's thumb. In this subsection, we sketch some graphs of Mandelbrot set at different input parameters for $M, M^{*}$, and $K$-iterative methods by using Algorithm 2 and compare the images of Mandelbrot set for proposed methods.

Example 4. In this example, we visualize some graphs of Mandelbrot sets for a polynomial $Q(z)=z^{2}+a_{0}$, where $a_{0} \in \mathbb{C}$ in the orbits of $M, M^{*}$, and $K$-iterative methods, 


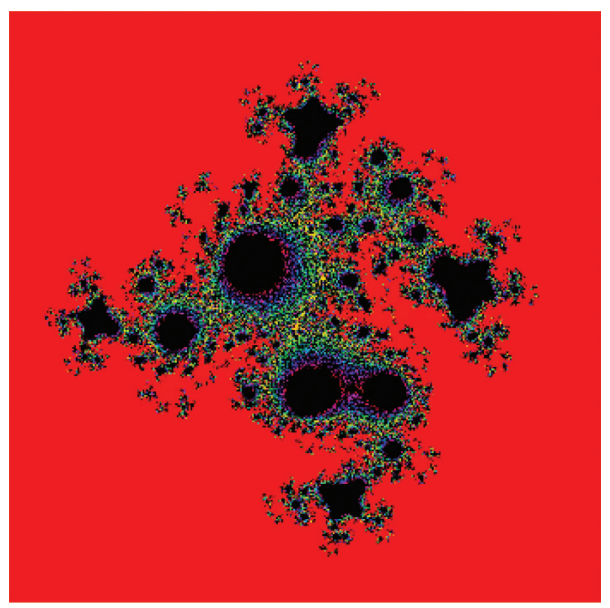

Figure 16: Julia set for $Q(z)$ with $n=4$ in $M$-orbit.

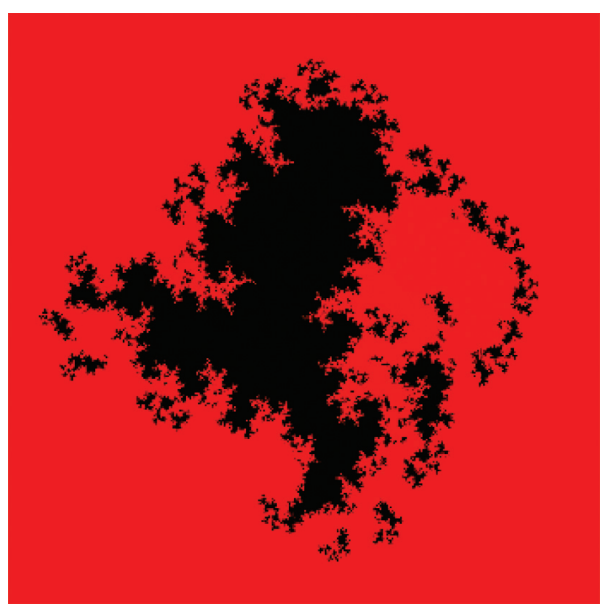

FIgURE 17: Julia set for $Q(z)$ with $n=4$ in $M^{*}$-orbit.

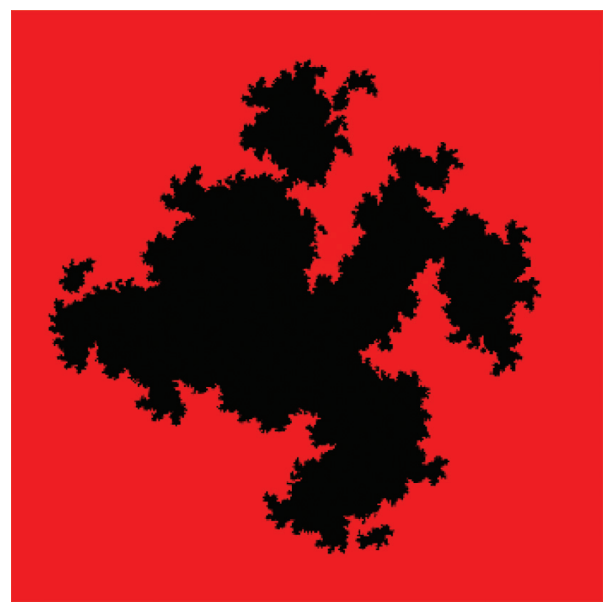

Figure 18: Julia set for $Q(z)$ with $n=4$ in $K$-orbit.

respectively. The input parameters for the graphs in Figures $19-21$ are $a=0.01, \quad b=0.9, \quad$ and $A=[-2,0.55] \times[-1.5,1.5]$. Figures $19-21$ are quadratic Mandelbrot sets via $M, M^{*}$, and $K$-iterative methods, respectively. The shapes of bulbs in each image are different. The images in Figures 19 and 20 relatively resemble classical Mandelbrot set, but Figure 21 is slightly different in shape. The main body or primary part of the images contains a large 
Input: $Q_{a_{0}}=z^{p}+a_{0}$-a complex polynomial, $A$-covered area, $\left.M=1000, a, b \in(0,1]\right)$-involved parameters, coloursmap $[0 . . h-$ 1] with $h$ colours.

Output: sketched Mandelbrot set.
(1) for $a_{0} \in A$ do
(2) $R$-convergence condition for proposed method
(3) $m=0$
(4) $z_{0}$-initial guess for $Q_{a_{0}}$
(5) while $m \leq K$ do
(6) Proposed iterative method
(7) if $\left|z_{m+1}\right|>R$ then
(8) break
(9) $m=m+1$
(10) $\quad i=\lfloor(h-1) m / M\rfloor$
(11) colour $a_{0}$ with coloursmap [i]

Algorithm 2: Pseudocode for Mandelbrot set.

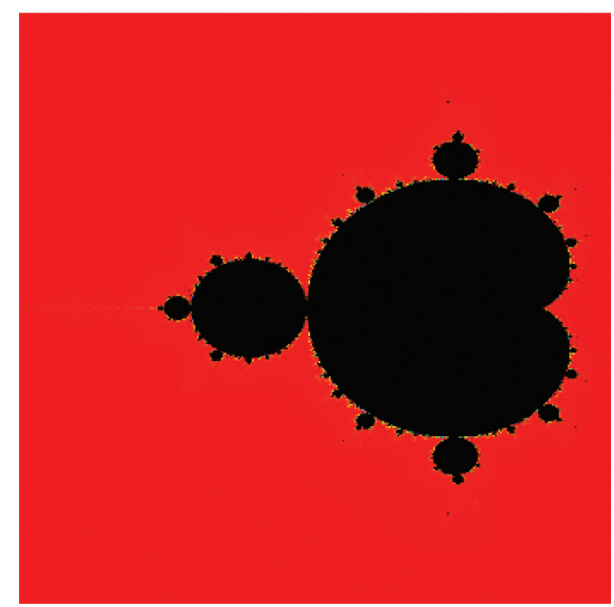

Figure 19: Mandelbrot set for $Q(z)$ with $n=2$ in $M$-orbit.

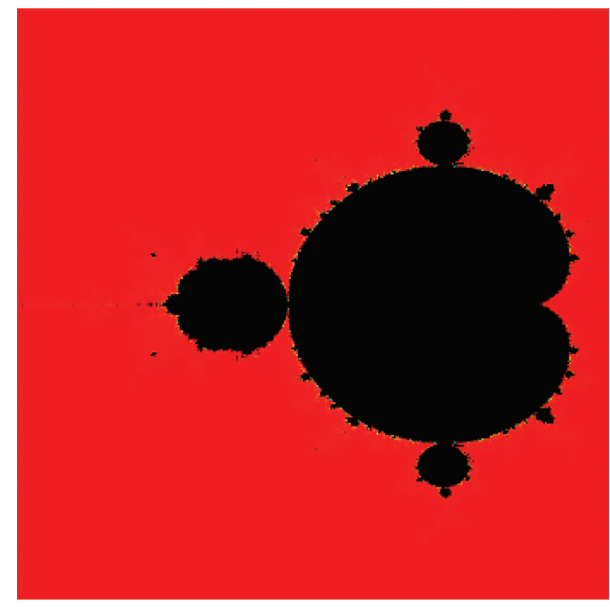

FIGURE 20: Mandelbrot set for $Q(z)$ with $n=2$ in $M^{*}$-orbit.

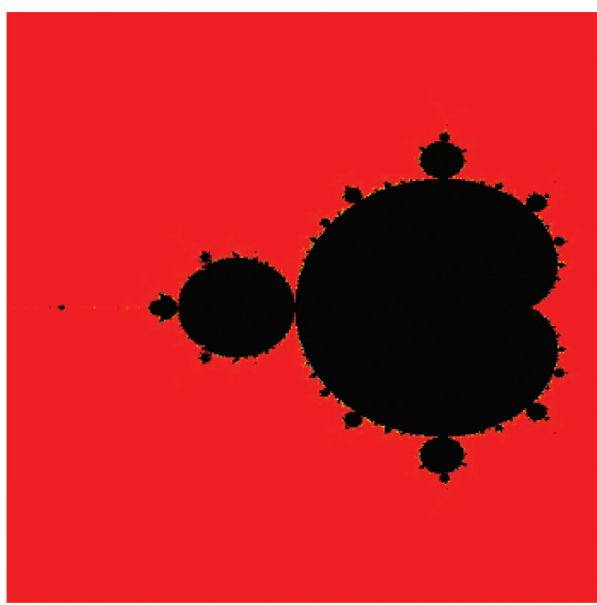

FIGURE 21: Mandelbrot set for $Q(z)$ with $n=2$ in $K$-orbit.

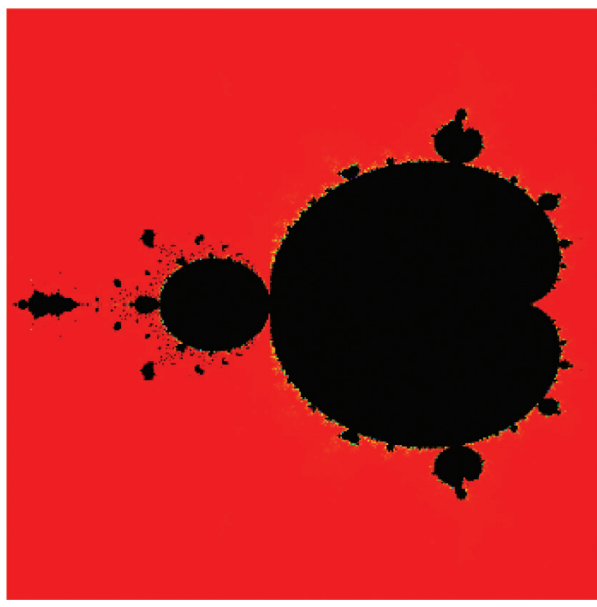

FIGURE 22: Mandelbrot set for $Q(z)$ with $n=2$ in $M$-orbit. 


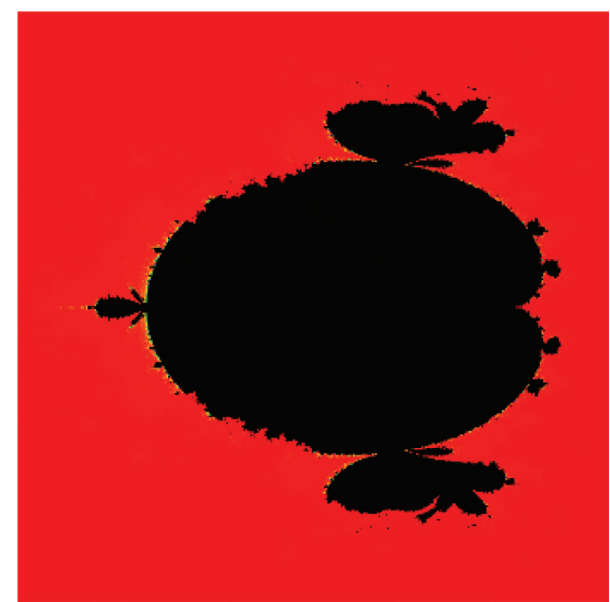

Figure 23: Mandelbrot set for $Q(z)$ with $n=2$ in $M^{*}$-orbit.

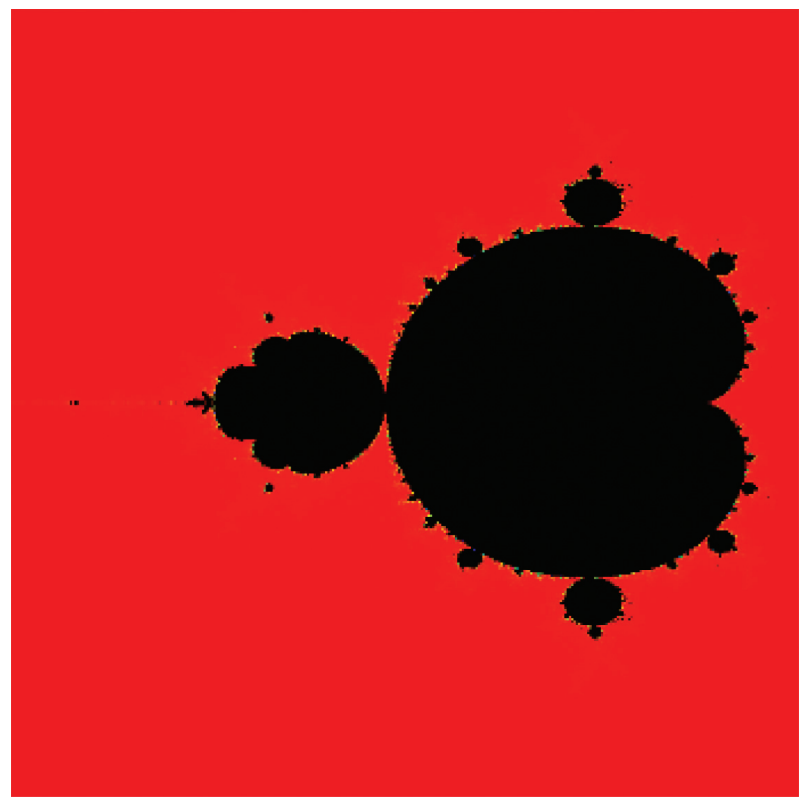

FIgURe 24: Mandelbrot set for $Q(z)$ with $n=2$ in $K$-orbit.

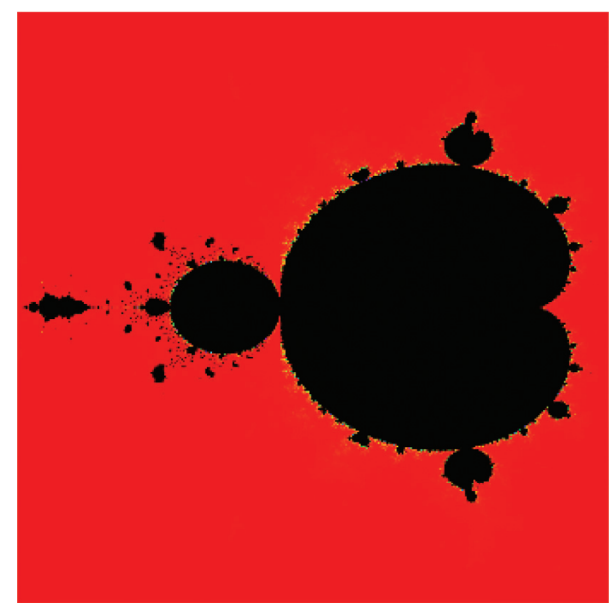

Figure 25: Mandelbrot set for $Q(z)$ with $n=2$ in $M$-orbit.

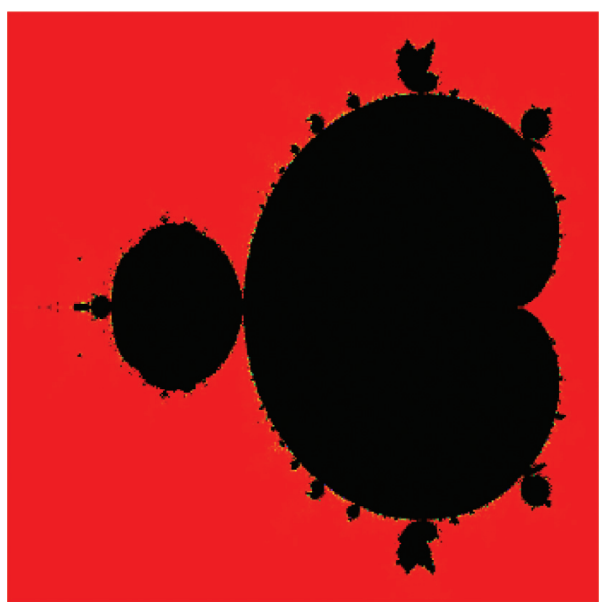

Figure 26: Mandelbrot set for $Q(z)$ with $n=2$ in $M^{*}$-orbit.

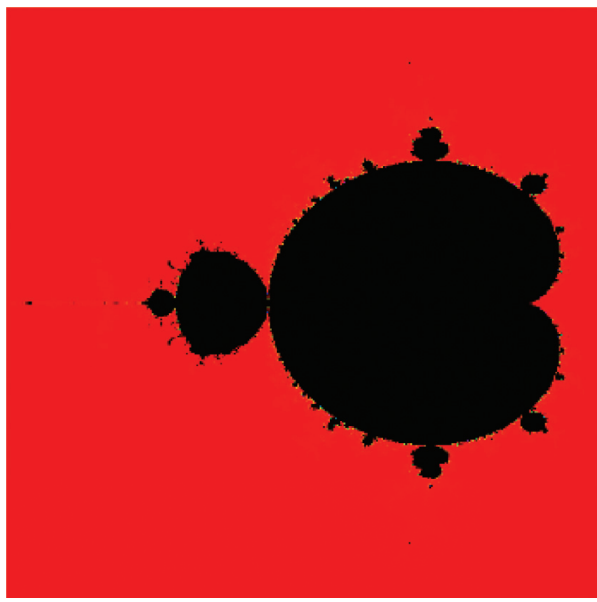

Figure 27: Mandelbrot set for $Q(z)$ with $n=2$ in $K$-orbit.

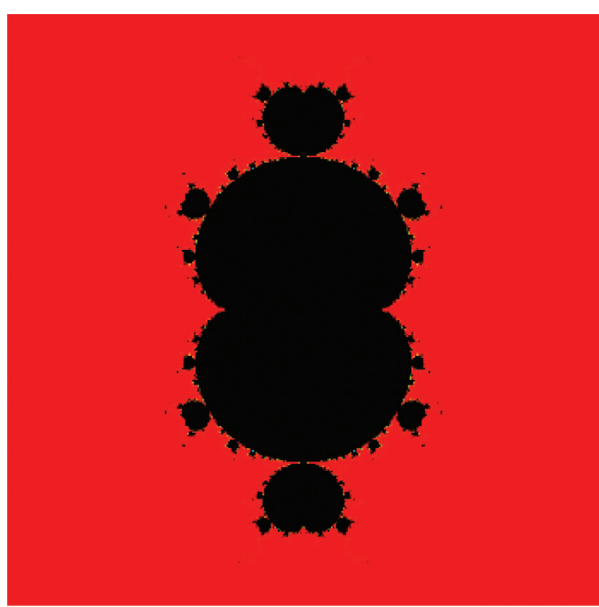

Figure 28: Mandelbrot set for $Q(z)$ with $n=3$ in $M$-orbit.

number of bulbs in different sizes, but if we magnify any bulb of image, it reflects the shape of whole image. In the generation of graphs in Figures 22-24, we change input 


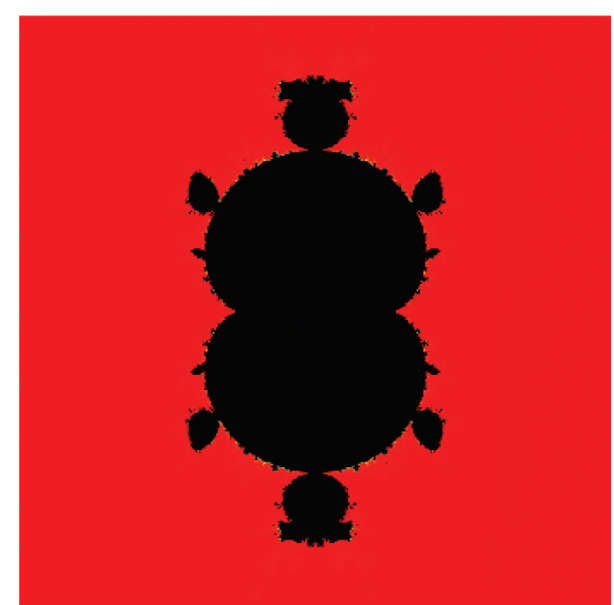

Figure 29: Mandelbrot set for $Q(z)$ with $n=3$ in $M^{*}$-orbit.

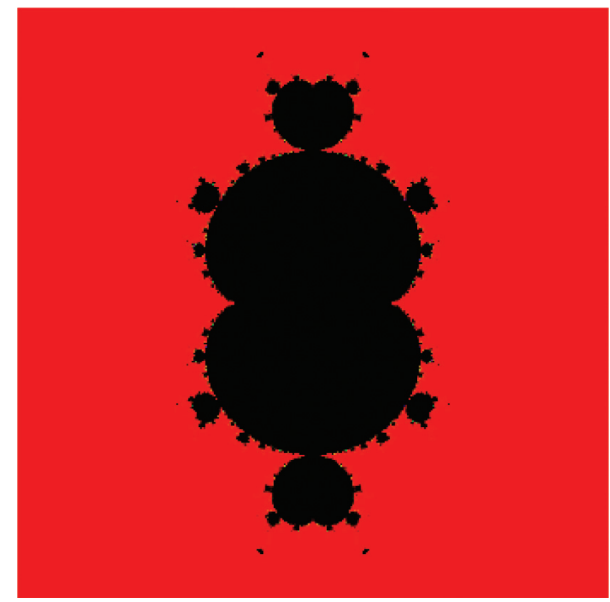

Figure 30: Mandelbrot set for $Q(z)$ with $n=3$ in $K$-orbit.

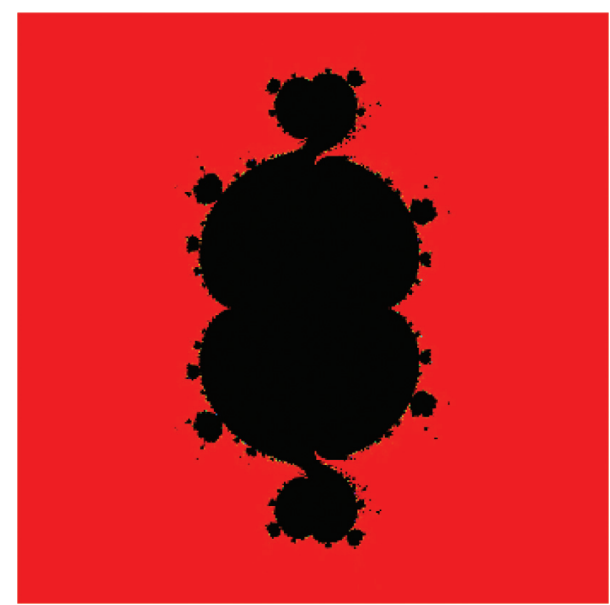

FIgURE 31: Mandelbrot set for $Q(z)$ with $n=3$ in $M$-orbit.

parameters $a$ and $b$. We observe that, for every method, images are completely different in shapes. Also the graphs in Figures 22 and 24 have input parameters $a=0.1, b=0.5$, and $A=[-2,0.55] \times[-1.5,1.5]$, but the graph in Figure 23 has input parameters $a=0.1, \quad b=0.5$, and

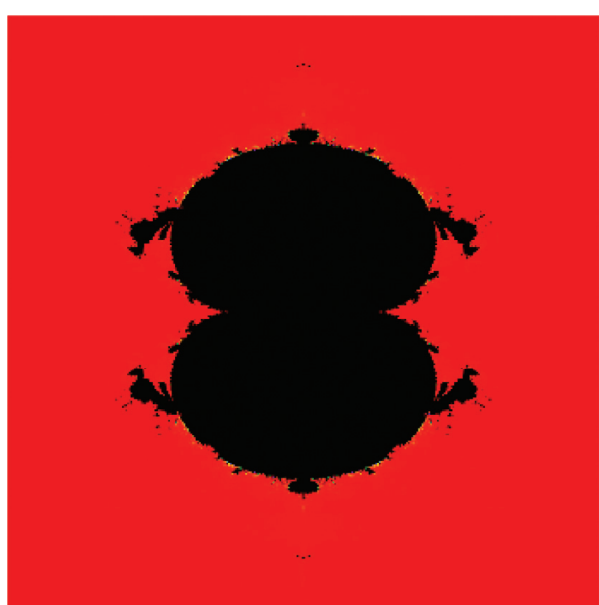

Figure 32: Mandelbrot set for $Q(z)$ with $n=3$ in $M^{*}$-orbit.

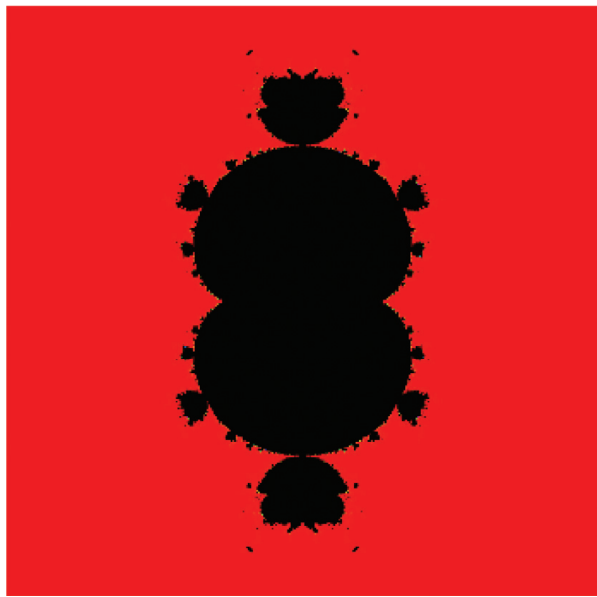

Figure 33: Mandelbrot set for $Q(z)$ with $n=3$ in $K$-orbit.

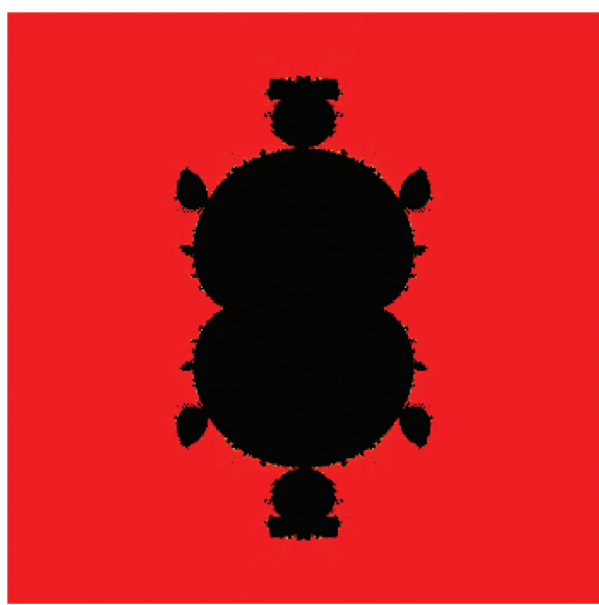

FIgURE 34: Mandelbrot set for $Q(z)$ with $n=3$ in $M$-orbit.

$A=[-3,0.85] \times[-2.5,2.5]$. The large bulbs in Figure 23 are like the wings of fish, and also the image covered a large area compared to images in Figures 22 and 24. In Figures 25-27, we again change the parameters $a$ and $b$ as $a, b=0.9$. All images have the same area as $A=[-2,0.55] \times[-1.5,1.5]$ but 


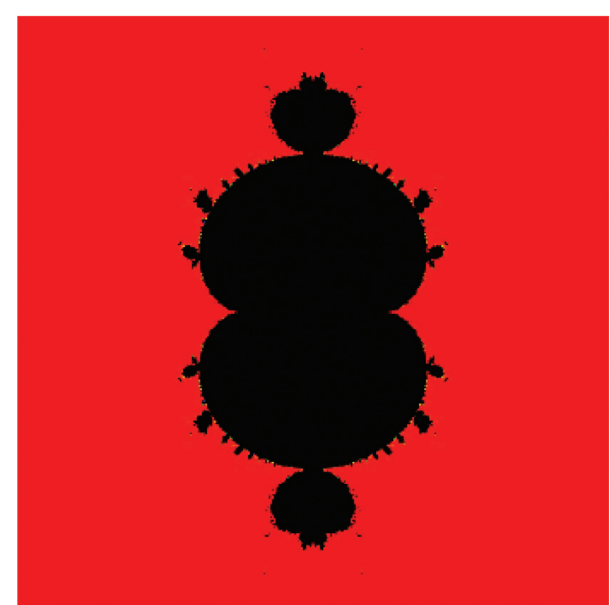

Figure 35: Mandelbrot set for $Q(z)$ with $n=3$ in $M^{*}$-orbit.

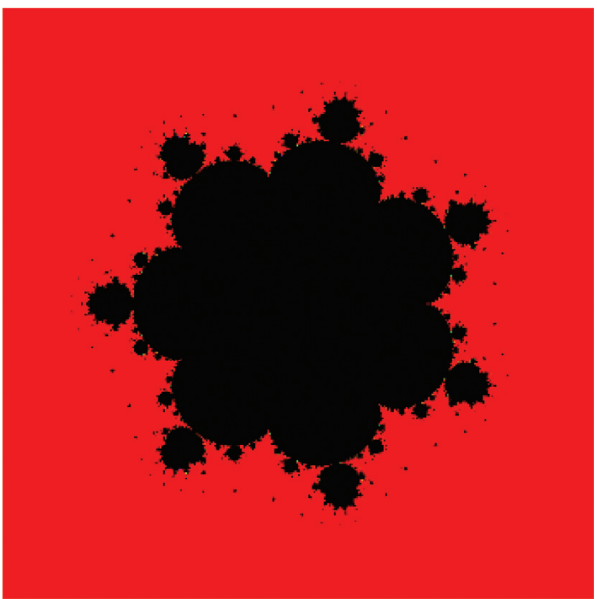

Figure 36: Mandelbrot set for $Q(z)$ with $n=8$ in $M$-orbit.

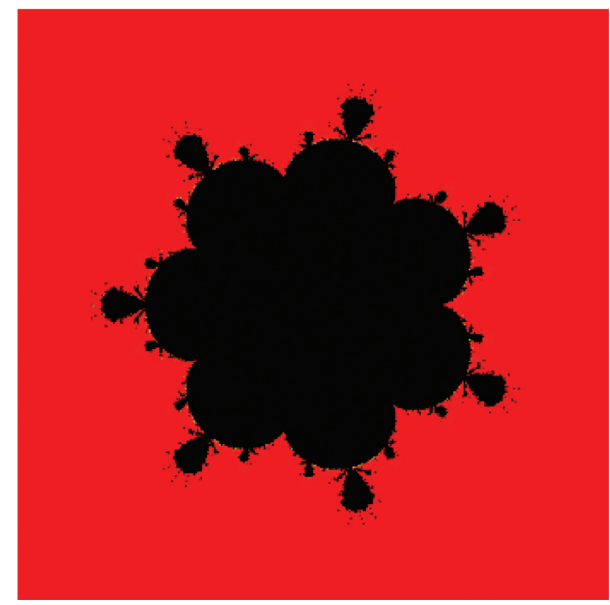

FIgURE 37: Mandelbrot set for $Q(z)$ with $n=8$ in $M^{*}$-orbit.

different in shapes primary (i.e., main cardioid) and secondary (i.e., bulbs on cardioid) parts.

Example 5. In this example, we visualize some graphs of Mandelbrot sets for a polynomial $Q(z)=z^{3}+a_{0}$, where

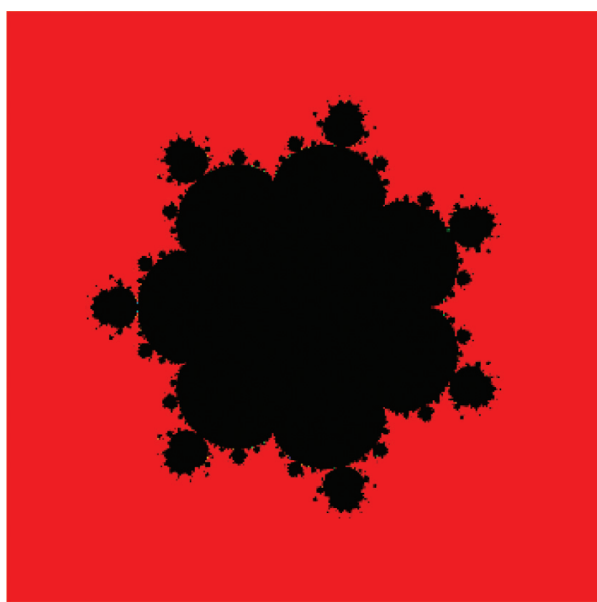

Figure 38: Mandelbrot set for $Q(z)$ with $n=8$ in $K$-orbit.

$a_{0} \in \mathbb{C}$ in the orbits of $M, M^{*}$, and $K$-iterative methods, respectively. From Figures 28-35, we perceive that each image has 2 cardioids, 2 large bulbs, and 4 small bulbs symmetry along $y$-axis. The shape of bulbs for each iterative method is different. The inputs for each cubic Mandelbrot set are as follows:

Figures 28-30 input parameters are $a=0.01, b=0.9$, and $A=[-1.5,1.5]^{2}$

Figures 31-33 input parameters are $a=0.1, b=0.5$, and $A=[-1.5,1.5]^{2}$

Figures 34-35 input parameters are $a=0.9, b=0.9$, and $A=[-1.5,1.5]^{2}$

Example 6. The last example demonstrates the ochto Mandelbrot sets for a polynomial $Q(z)=z^{8}+a_{0}$, where $a_{0} \in \mathbb{C}$ in the orbits of $M, M^{*}$, and $K$-iterative methods, respectively. All images for the graphs in Figures 36-38 have the same inputs as $a=0.01, b=0.9$, and $A=[-1.5,1.5]^{2}$. We notice that 7 large bulbs appear on the main body of each ochto Mandelbrot set. The shape of bulbs for each method is also different in images.

\section{Conclusions}

We analyzed $M, M^{*}$, and $K$-iterative methods in the generation of Julia and Mandelbrot sets. We established some convergence conditions for the orbits of $M, M^{*}$, and $K$ iterative methods, respectively. We used the established convergence conditions in algorithms to sketch some Julia and Mandelbrot sets. Fascinating Julia and Mandelbrot sets were generated for different input parameters and compared the images. We observed that, for each proposed method, image is slightly different in shape from other two methods. Furthermore, we noticed that, for a very small change in any input parameter, the images drastically changed. Moreover, we concluded that the complex graphs of Julia and Mandelbrot sets generated in this research were the application of fractal geometry. 


\section{Data Availability}

Data are included within this paper.

\section{Conflicts of Interest}

The authors declare that they have no conflicts of interest.

\section{Authors' Contributions}

All authors have contributed equally.

\section{Acknowledgments}

This research was partitively supported by the funds of University of Lahore, Lahore, Pakistan.

\section{References}

[1] S. Salawu, M. Sobamowo, and O. Sadiq, "Dynamic analysis of non-homogenous varying thickness rectangular plates resting on pasternak and winkler foundations," Engineering and Applied Science Letters, vol. 3, pp. 1-20, 2020.

[2] X. Zhang, L. Wang, Z. Zhou, and Y. Niu, "A chaos-based image encryption technique utilizing hilbert curves and H-fractals," IEEE Access, vol. 7, pp. 74734-74746, 2019.

[3] W. R. Watts and D. Walker, "Kinetic studies and effects of anions on creatine phosphokinase from skeletal muscle of rhesus monkey (Macaca mulatta)," Biochimica et biophysica acta, vol. 410, no. 1, pp. 99-114, 1975.

[4] N. Cohen, "Fractal antenna applications in wireless telecommunications," in Proceedings of the Professional Program Proceedings. Electronic Industries Forum of New England, pp. 43-49, IEEE, Boston, MA, USA, May 1997.

[5] W. J. Krzysztofik, "Fractals in antennas and metamaterials applications," in Fractal Analysis-Applications in Physics, Engineering and Technology, F. Brambila, Ed., INTECH Open Science, Rijeka, Croatia, pp. 978-953, 2017.

[6] F. Orsucci, Complexity Science, Living Systems, and Reflexing Interfaces: New Models and Perspectives: New Models and Perspectives, IGI Global, Hershey, PA, USA, 2012.

[7] M. Bouallala, E. Essoufi, and A. Zafrar, "Analysis and numeric of mixed approach for frictional contact problem in electroelasticity," Open Journal of Mathematical Analysis, vol. 4, no. 1, pp. 20-37, 2020.

[8] Z. Bekri and S. Benaicha, "Positive solutions for boundary value problem of sixth-order elastic beam equation," Open Journal of Mathematical Analysis, vol. 4, no. 1, pp. 9-17, 2020.

[9] A. D. Oguz and F. S. Topal, "Existence result for a singular semipositone dynamic system on time scales," Open Journal of Mathematical Analysis, vol. 4, no. 1, pp. 86-97, 2020.

[10] B. B. Mandelbrot, The Fractal Geometry of Nature, Vol. 2, WH Freeman, New York, NY, USA, 1982.

[11] K. Gdawiec and A. A. Shahid, "Fixed point results for the complex fractal generation in the S-iteration orbit with s-convexity," Open Journal of Mathematical Analysis, vol. 2, pp. 52-72, 2018.

[12] A. Lakhtakia, V. V. Varadan, R. Messier, and V. K. Varadan, "On the symmetries of the Julia sets for the process $\mathrm{z} \Rightarrow \mathrm{zp}+\mathrm{c}$," Journal of Physics A: Mathematical and General, vol. 20, no. 11, pp. 3533-3535, 1987.

[13] P. Blanchard, R. L. Devaney, A. Garijo, and E. D. Russell, "A generalized version of the Mcmullen domain," International
Journal of Bifurcation and Chaos, vol. 18, no. 8, pp. 2309-2318, 2008.

[14] T. Kim, "Quaternion Julia set shape optimization," Computer Graphics Forum, vol. 34, no. 5, pp. 167-176, 2015.

[15] V. Drakopoulos, N. Mimikou, and T. Theoharis, "An overview of parallel visualisation methods for Mandelbrot and Julia sets," Computers \& Graphics, vol. 27, no. 4, pp. 635-646, 2003.

[16] Y. Sun, L. Chen, R. Xu, and R. Kong, "An image encryption algorithm utilizing Julia sets and Hilbert curves," PLoS One, vol. 9, no. 1, Article ID e84655, 2014.

[17] M. Rani and V. Kumar, "Superior Julia set," Research in Mathematical Education, vol. 8, no. 4, pp. 261-277, 2004.

[18] M. Rani and V. Kumar, "Superior Mandelbrot set," Research in Mathematical Education, vol. 8, no. 4, pp. 279-291, 2004.

[19] M. Rani and R. Agarwal, "Effect of stochastic noise on superior Julia sets," Journal of Mathematical Imaging and Vision, vol. 36, no. 1, pp. 63-68, 2010.

[20] A. Nagi, M. Rani, and R. Chugh, "Julia sets and Mandelbrot sets in Noor orbit," Applied Mathematics and Computation, vol. 228, pp. 615-631, 2014.

[21] S. Kang, W. Nazeer, M. Tanveer, and A. Shahid, "New fixed point results for fractal generation in Jungck Noor orbit with s-convexity," Journal of Function Spaces, vol. 2015, Article ID 963016, 7 pages, 2015.

[22] M. Tanveer, S. M. Kang, W. Nazeer, and Y. C. Kwun, "New tricorns and multicorns antifractals in jungck mann orbit," International Journal of Pure and Applied Mathematics, vol. 111, no. 2, pp. 287-302, 2016.

[23] K. Goyal and B. Prasad, "Dynamics of iterative schemes for quadratic polynomial," AIP Conference, vol. 9, no. 2, pp. 149-153, 2001.

[24] M. Kumari, A. Nagi, and C. Renu, "New Julia and Mandelbrot sets for a new faster iterative process," International Journal of Pure and Applied Mathematics, vol. 107, no. 1, pp. 161-177, 2016.

[25] W. Nazeer, S. M. Kang, M. Tanveer, and A. A. Shahid, "Fixed point results in the generation of Julia and Mandelbrot sets," Journal of Inequalities and Applications, vol. 2015, no. 1, p. 298, 2015.

[26] S. M. Kang, A. Rafiq, A. Latif, A. A. Shahid, and Y. C. Kwun, "Tricorns and multicorns of S-iteration scheme," Journal of Function Spaces, vol. 2015, Article ID 417167, 7 pages, 2015.

[27] Y. C. Kwun, M. Tanveer, W. Nazeer, M. Abas, and S. M. Kang, "Fractal generation in modified Jungck-S orbit," IEEE Access, vol. 7, pp. 35060-35071, 2019.

[28] Y. C. Kwun, M. Tanveer, W. Nazeer, K. Gdawiec, and S. M. Kang, "Mandelbrot and julia sets via Jungck-Cr iteration with S-convexity," IEEE Access, vol. 7, 2019.

[29] D. Li, M. Tanveer, W. Nazeer, and X. Guo, "Boundaries of filled Julia sets in generalized jungck mann orbit," IEEE Access, vol. 7, 2019.

[30] X. Li, M. Tanveer, M. Abbas, M. Ahmad, Y. C. Kwun, and J. Liu, “"Fixed point results for fractal generation in extended jungck-sp orbit,” IEEE Access, vol. 7, 2019.

[31] E. Zinab, K. Koyas, K. Koyas, and A. Girma, "A fixed point theorem for generalized weakly contractive mappings in $\mathrm{b}$ -metric spaces," Open Journal of Mathematical Sciences, vol. 4, no. 1, pp. 1-8, 2020.

[32] I. Ahmad, M. Ahmad, and M. Ahmad, "An implicit viscosity technique of nonexpansive mapping in CAT(0) spaces," Open Journal of Mathematical Analysis, vol. 1, no. 1, pp. 1-12, 2017.

[33] M. Tesfaye, K. Koyas, K. Koyas, and S. Gebregiorgis, "A coupled fixed point theorem for maps satisfying rational type contractive condition in dislocated quasi b-metric space," 
Open Journal of Mathematical Sciences, vol. 4, no. 1, pp. 27-33, 2020.

[34] S. Kausar, M. Asif, and M. Munir, "Non-convex hybrid method corresponding to karakaya iterative process," Open Journal of Mathematical Analysis, vol. 2, no. 1, 2020.

[35] A. Perveen, S. Kausar, S. Kausar, and W. Nazeer, "Strong convergence theorems of common fixed points for a uniformly closed asymptotically family of countable quasi-lipschitz mappings in hilbert spaces," Open Journal of Mathematical Analysis, vol. 3, no. 1, pp. 1-6, 2019.

[36] S. F. A. Naqvi, M. S. Khan, and M. S. Khan, "On the viscosity rule for common fixed points of two nonexpansive mappings in hilbert spaces," Open Journal of Mathematical Sciences, vol. 1, no. 1, pp. 111-125, 2017.

[37] V. V. Strotov, S. A. Smirnov, S. E. Korepanov, and A. V. Cherpalkin, "Object distance estimation algorithm for real-time fpga-based stereoscopic vision system," in Proceedings of the High-Performance Computing in Geoscience and Remote Sensing VIII, vol. 10792, p. 107920A, September 2018.

[38] O. Khatib, "Real-time obstacle avoidance for manipulators and mobile robots," in Autonomous Robot Vehicles, pp. 396-404, Springer, Berlin, Germany, 1986.

[39] J. Barrallo and D. M. Jones, "Coloring algorithms for dynamical systems in the complex plane," in Visual Mathematics, vol. 1, no. 4, Mathematical Institute SASA, Belgrade, Serbia, 1999.

[40] M. Barnsley, Fractals Everywhere, Academic Press, Boston, MA, USA, 2nd edition, 1993.

[41] R. Devaney, A First Course in Chaotic Dynamical Systems: Theory and Experiment, Addison-Wesley, New York, NY, USA, 1992.

[42] L. Xiangdong, Z. Zhiliang, W. Guangxing, and Z. Weiyong, "Composed accelerated escape time algorithm to construct the general Mandelbrot set," Fractals, vol. 9, no. 2, pp. 149$153,2001$.

[43] N. Hussain, K. Ullah, and M. Arshad, "Fixed point Approximation of suzuki generalized nonexpansive mappings via new faster iteration process," vol. 7, 2018, http://arxiv.org/ abs/1802.09888. 